\title{
ELASTODYNAMIC CRACK PROBLEMS \\ IN AN ANISOTROPIC MEDIUM \\ THROUGH A COMPLEX VARIABLE APPROACH*
}

BY

A. PIVA

University of Bologna, Italy

\begin{abstract}
The complex variable approach is used to obtain closed-form solutions to elastodynamic crack problems in a strip of anisotropic elastic medium, with one plane of symmetry, under antiplane shear stress. The two problems examined involve a finite-length crack propagating in an infinitely long strip of finite width when the edges are subjected either to constant displacements or shearing stresses. The special cases corresponding to either an orthotropic medium or an isotropic medium are recovered.
\end{abstract}

1. Introduction. In recent papers [1, 2], the complex variable approach has been used to obtain closed-form solutions to elastodynamic crack problems in a homogeneous and isotropic elastic strip under antiplane shear stress.

The problems analyzed are the classical ones of a finite-length crack moving at constant velocity in an infinitely long strip of finite width with the lateral boundaries subjected to either displacements or shearing stresses. Both problems have been reduced to half-plane boundary value problems, solved by the Keldysh-Sedov method as explained in [3]. This kind of problem has also been studied in [4] by an integral transform technique and recently extended in [5] to an orthotropic layer.

In this paper the elastodynamic crack problems mentioned above are solved for a strip made of an anisotropic medium with one plane of symmetry. After transformation of the basic equations, the complex variable approach is used to obtain closed-form expressions for the stress field as well as the stress intensity factors at the crack tip. The results obtained in $[4,5]$ are recovered as particular cases.

2. Basic equations and formulation of problems. Consider an infinitely long strip of width $2 h$ of homogeneous anisotropic elastic material referred to a fixed Cartesian coordinate system $0 x_{1} x_{2} x_{3}$. The strip contains a crack of length $2 l$ traveling with constant

\footnotetext{
* Received January 8, 1985.
} 
velocity $c$ in the positive $x_{1}$-direction. Supposing that the medium contains a twofold rotation axis parallel to the $x_{3}$-axis, the elastic constants are given in the following form [6]:

$$
\left[\begin{array}{cccccc}
C_{11} & C_{12} & C_{13} & 0 & 0 & C_{16} \\
& C_{22} & C_{23} & 0 & 0 & C_{26} \\
& & C_{33} & 0 & 0 & C_{36} \\
& & & C_{44} & C_{45} & 0 \\
& & & & C_{55} & 0 \\
& & & & & C_{66}
\end{array}\right] .
$$

Assuming an antiplane shear field, the elastic state is independent of $x_{3}$ and the stress components are

$$
\begin{aligned}
& \sigma_{13}=C_{45} \frac{\partial u_{3}}{\partial x_{2}}+C_{55} \frac{\partial u_{3}}{\partial x_{1}}, \\
& \sigma_{23}=C_{44} \frac{\partial u_{3}}{\partial x_{2}}+C_{45} \frac{\partial u_{3}}{\partial x_{1}},
\end{aligned}
$$

where $u_{3}=u_{3}\left(x_{1}, x_{2}, \tau\right)$ is the only nonvanishing displacement component which satisfies the following equation of motion:

$$
C_{55} \frac{\partial^{2} u_{3}}{\partial x_{1}^{2}}+2 C_{45} \frac{\partial^{2} u_{3}}{\partial x_{1} \partial x_{2}}+C_{44} \frac{\partial^{2} u_{3}}{\partial x_{2}^{2}}=\rho \frac{\partial^{2} u_{3}}{\partial \tau^{2}},
$$

$\rho$ being the density of the material. Assuming $u_{3}=u_{3}\left(x_{1}-c \tau, x_{2}\right)$ and introducing the transformation

$$
X_{1}=x_{1}-c \tau, \quad X_{2}=s x_{2}, \quad \tau=\tau,
$$

where $s^{2}=1-c^{2} / \bar{c}^{2}, \bar{c}^{2}=C_{55} / \rho$, Eq. (2.4) becomes

$$
\frac{\partial^{2} u_{3}}{\partial X_{1}^{2}}+2 \beta \frac{\partial^{2} u_{3}}{\partial X_{1} \partial X_{2}}+\alpha \frac{\partial^{2} u_{3}}{\partial X_{2}^{2}}=0
$$

where $\alpha=C_{44} / C_{55}, \beta=C_{45} / s C_{55}$ (in what follows, it will be assumed that $\beta>0$, $\alpha>\beta^{2}$ ).

By setting

$$
\xi=X_{2}-\beta X_{1}, \quad \eta=\sqrt{\Delta} X_{1},
$$

where $\Delta=\alpha-\beta^{2}$, Eq. (2.6) reduces to the canonical form

$$
\frac{\partial^{2} u_{3}}{\partial \xi^{2}}+\frac{\partial^{2} u_{3}}{\partial \eta^{2}}=0
$$

At this step it will be convenient to apply the conformal transformation

$$
w=u+i v=e^{i \theta}(\eta+i \xi),
$$


where $\theta=\tan ^{-1}(\beta / \sqrt{\Delta})$, which maintains the harmonic feature of $u_{3}$ in the $(u, v)$ plane. In view of (2.5), (2.7), and (2.9), the stress-strain Eqs. (2.2) and (2.3) become

$$
\begin{gathered}
\sigma_{13}=\frac{C_{55} \beta}{\sqrt{\alpha}}\left[\left(\frac{\alpha-\beta^{2} s^{2}}{\beta}\right) \frac{\partial u_{3}}{\partial u}+s^{2} \sqrt{\Delta} \frac{\partial u_{3}}{\partial v}\right], \\
\sigma_{23}=s \sqrt{\alpha \Delta} C_{55} \frac{\partial u_{3}}{\partial v} .
\end{gathered}
$$

It is the purpose of this paper to present the solution for the following problems:

Problem I. The boundaries at $X_{2}= \pm h s$ are clamped and displaced in the opposite $X_{3}$-directions by an amount $u_{0}$ which allows antiplane crack motion in the positive $X_{1}$-direction. The boundary value problem is formulated as follows:

$$
\begin{gathered}
u_{3}\left(X_{1}, \pm h s\right)= \pm u_{0}, \quad\left|X_{1}\right|<\infty, \\
\alpha \frac{\partial u_{3}}{\partial X_{2}}+\beta \frac{\partial u_{3}}{\partial X_{1}}=0, \quad\left|X_{1}\right|<l, X_{2}=0, \\
u_{3}\left(X_{1}, 0\right)=0, \quad\left|X_{1}\right|>l,
\end{gathered}
$$

where $u_{3}\left(X_{1}, X_{2}\right)$ is the solution of Eq. (2.6).

Problem II. The boundaries at $X_{2}= \pm h s$ are sheared by a constant stress $\sigma_{0}$. The problem consists of solving Eq. (2.6) together with the following boundary conditions:

$$
\begin{gathered}
\alpha \frac{\partial u_{3}}{\partial X_{2}}+\beta \frac{\partial u_{3}}{\partial X_{1}}=\frac{\sigma_{0}}{s C_{55}}, \quad\left|X_{1}\right|<\infty, X_{2}= \pm h s, \\
\alpha \frac{\partial u_{3}}{\partial X_{2}}+\beta \frac{\partial u_{3}}{\partial X_{1}}=0, \quad\left|X_{1}\right|<l, X_{2}=0, \\
u_{3}\left(X_{1}, 0\right)=0, \quad\left|X_{1}\right|>l .
\end{gathered}
$$

3. Solution of Problem I. The transformations (2.7) and (2.9) reduce Problem I to finding the harmonic function $u_{3}(u, v)$ satisfying the following boundary conditions:

$$
\begin{gathered}
u_{3}\left(u, \pm v_{0}\right)= \pm u_{0}, \quad|u|<\infty \\
\frac{\partial u_{3}}{\partial v}=0, \quad|u|<l_{1}, v=0, \\
u_{3}(u, 0)=0, \quad|u|>l_{1},
\end{gathered}
$$

where

$$
v_{0}=h s \sqrt{\Delta / \alpha}, \quad l_{1}=l \sqrt{\alpha} .
$$

Applying the same method as in [1, 2], the problem (3.1) reduces to a half-plane boundary value problem, solved by the Keldish-Sedov technique [3] in terms of the complex function

$$
\Phi(\omega)=\frac{u_{0}}{v_{0} a} \ln \left[\frac{\omega+1}{\sqrt{1-\delta^{2}}+\sqrt{\omega^{2}-\delta^{2}}}\right],
$$

where $a=\pi / 2 v_{0}, \delta=\tanh \left(a l_{1}\right)$, and $\omega=\tanh (a w)$. 
Regarding the derivatives of the displacement function, the following expressions hold:

$$
\begin{gathered}
\frac{\partial u_{3}}{\partial u}=a \operatorname{Im}\left[\left(1-\omega^{2}\right) \Phi^{\prime}(\omega)\right] \\
\frac{\partial u_{3}}{\partial v}=\frac{u_{0}}{v_{0}}+a \operatorname{Re}\left[\left(1-\omega^{2}\right) \Phi^{\prime}(\omega)\right]
\end{gathered}
$$

Hence, from (2.10) and (2.11) the stress components may be obtained. In particular, Eq. (2.11) gives

$$
\sigma_{23}=\frac{\alpha C_{55} u_{0}}{h} \operatorname{Re}\left[\frac{\sinh (a w)}{\left(\sinh ^{2}(a w)-\sinh ^{2}\left(a l_{1}\right)\right)^{1 / 2}}\right] .
$$

It is of interest in fracture mechanics to have the stress intensity factor at the tip $(l, 0)$, defined as

$$
k_{1}=\lim _{X_{1} \rightarrow l_{+}} \sqrt{2\left(X_{1}-l\right)} \sigma_{23}\left(X_{1}, 0\right)
$$

which gives

$$
K_{1}=\frac{\alpha C_{55} u_{0}}{h} \sqrt{\frac{\tanh (a \sqrt{\alpha} l)}{a \sqrt{\alpha}}} .
$$

In the orthotropic case $C_{45}=0$ and Eq. (3.7) is in agreement with the corresponding one obtained in [5]. In the isotropic case $C_{45}=C_{55}=\mu$ (shear modulus), $C_{45}=0$, and the result of [4] is recovered.

4. Solution of Problem II. The transformations (2.7) and (2.9) reduce Problem II to finding the harmonic function $u_{3}(u, v)$ satisfying the following boundary conditions:

$$
\begin{gathered}
\frac{\partial u_{3}}{\partial v}\left(u, \pm v_{0}\right)=p_{0}, \quad|u|<\infty, \\
\frac{\partial u_{3}}{\partial v}(u, 0)=0, \quad|u|<l_{1}, \\
u_{3}(u, 0)=0, \quad|u|>l_{1},
\end{gathered}
$$

where $p_{0}=\sigma_{0} /\left(s C_{55} \sqrt{\alpha \Delta}\right)$.

Proceeding again as in $[1,2,3]$ the solution of problem (4.1) is obtained in terms of the complex function

$$
\Psi(\omega)=\frac{2 p_{0} \omega\left(1-\delta^{2}\right)}{\pi \sqrt{\left(\omega^{2}-1\right)\left(\omega^{2}-\delta^{2}\right)}}\left[\Pi\left(\gamma^{2}, \delta\right)-\mathrm{K}(\delta)\right],
$$

where $\mathrm{K}(\delta)$ is the complete elliptic integral of the first kind of modulus $\delta$ and $\Pi\left(\gamma^{2}, \delta\right)$ is the complete elliptic integral of the third kind of the same modulus and complex parameter $\gamma^{2}=\delta^{2}\left(1-\omega^{2}\right) /\left(\omega^{2}-\delta^{2}\right)$. The stress component (2.11) has the following formal expression:

$$
\sigma_{23}=\sigma_{0}\left\{1+\frac{2}{\pi}\left(1-\delta^{2}\right) \operatorname{Im}\left[\frac{\omega}{\sqrt{\left(\omega^{2}-1\right)\left(\omega^{2}-\delta^{2}\right)}}\left(\Pi\left(\gamma^{2}, \delta\right)-\mathrm{K}(\delta)\right)\right]\right\} .
$$


In particular, on $l<X_{1}<\infty$,

$$
\begin{array}{r}
\sigma_{23}\left(X_{1}, 0\right)=\sigma_{0}\left\{1-\frac{\sinh \left(2 a \sqrt{\alpha} X_{1}\right)}{\pi \cosh (a \sqrt{\alpha} l)\left(\sinh ^{2}\left(a \sqrt{\alpha} X_{1}\right)-\sinh ^{2}(a \sqrt{\alpha} l)\right)^{1 / 2}}\right. \\
\cdot\left[\Pi\left(\gamma_{1}^{2}, \tanh (a \sqrt{\alpha} l)-\mathrm{K}(\tanh (a \sqrt{\alpha} l))\right]\right\},
\end{array}
$$

where $\gamma_{1}^{2}=\sinh ^{2}(a \sqrt{\alpha} l) /\left(\left(\sinh ^{2}(a \sqrt{\alpha} l)-\sinh ^{2}\left(a \sqrt{\alpha} X_{1}\right)\right)\right.$, which implies the following expression for the stress intensity factor at the tip $(l, 0)$ :

$$
K_{2}=\frac{2 \sigma_{0}}{\pi} \sqrt{\frac{\tanh (a \sqrt{\alpha} l)}{a \sqrt{\alpha}}} \mathrm{K}(\tanh (a \sqrt{\alpha} l)) .
$$

For an orthotropic material, Eqs. (4.4) and (4.5) are in agreement with those obtained in [5], apart from the additional constant term in (4.4). This disagreement probably arises from the fact that the superposition principle was not applied in [5]. The isotropic case is recovered as in [4] with the same comment.

Acknowledgment. This work was performed in the framework of the activities of the "Gruppo Nazionale per la Fisica Matematica," C.N.R.

\section{REFERENCES}

[1] R. J. Tait and T. B. Moodie, Complex variable methods and closed-form solutions to dynamic crack and punch problems in the classical theory of elasticity, Internat. J. Eng. Sci. 19, 221-229 (1981)

[2] R. J. Tait and T. B. Moodie, On a problem in the dynamic theory of cracks, Quart. Appl. Math. 38, 419-423 (1981)

[3] F. D. Gakhov, Boundary value problems, Pergamon, New York, 1966

[4] B. M. Singh, T. B. Moodie, and J. Haddow, Closed-form solutions for finite length crack moving in a strip under anti-plane shear stress, Acta Mech. 38, 99-109 (1981)

[5] H. T. Danyluk and B. M. Singh, Closed form solutions for a finite length crack moving in an orthotropic layer of finite thickness, Lett. Appl. Eng. Sci. 22, 637-644 (1984)

[6] S. G. Lekhnitskii, Anisotropic plates, Gordon \& Breach, Philadelphia, 1968 